\title{
Submitochondrial particles from Saccharomyces cerevisiae to screen compounds for antiprotozoal activity
} Carmen Streicher ${ }^{1}$, Gerlinde Wiesenberger ${ }^{2}$, Thomas Rosenau ${ }^{3}$ and Lars Gille*1

\author{
Address: ${ }^{1}$ Department of Natural Sciences; University of Veterinary Medicine Vienna, Austria, ${ }^{2}$ Max F. Perutz Laboratories, University of Vienna, \\ Austria and ${ }^{3}$ Department of Chemistry, University of Natural Resources and Applied Life Sciences, Vienna, Austria \\ Email: Lars Gille* - lars.gille@vu-wien.ac.at \\ * Corresponding author
}

from I3th Scientific Symposium of the Austrian Pharmacological Society (APHAR). Joint Meeting with the Austrian Society of Toxicology (ASTOX) and the Hungarian Society for Experimental and Clinical Pharmacology (MFT)

Vienna, Austria. 22-24 November 2007

Published: 14 November 2007

BMC Pharmacology 2007, 7(Suppl 2):A56 doi:10.1 I86/147I-2210-7-S2-A56

This abstract is available from: http://www.biomedcentral.com/I47I-22/0/7/S2/A56

(c) 2007 Streicher et al; licensee BioMed Central Ltd.

The development of atovaquone (ATQ)-resistant plasmodia strains suggests a need of new drugs for the mitochondrial $b_{1}$ complex as drug target in protozoa. It was the aim of the study to establish a model consisting of submitochondrial particles (SMP) from yeast (mimicking the protozoal drug target) and bovine heart for the screening of compounds. Saccharomyces cerevisiae wild-type strains (W303-1A, DBY747) were grown on YPD-media at $30^{\circ} \mathrm{C}$ for three days. After harvesting the cells their cell wall was digested by zymolyase and mitochondria were isolated. From these organelles SMP where prepared by sonication. The inhibition of the quinol:cyt c oxidoreductase activity catalyzed by the mitochondrial $\mathrm{bc}_{1}$ complex of yeast and bovine heart SMP was measured for a nonspecies-specific inhibitor stigmatellin and for the anti-malaria drug ATQ. $\mathrm{IC}_{50}$ values for stigmatellin in both SMP preparations confirmed its nonselectivity while ATQ inhibited the yeast complex activity about 16 times stronger than the activity of bovine heart SMP. This confirms the functionality of yeast SMP as model for protozoa. A new lead structure TMC2O demonstrated an $\mathrm{IC}_{50}$ value of $808 \mu \mathrm{M}$ and 112 $\mu \mathrm{M}$ in yeast and bovine heart SMP, respectively. This suggests that structural adjustments are required to increase the inhibition in yeast/protozoa. The yeast model in context with yeast strains mimicking mutated protozoa provides an efficient way to develop drugs for ATQ-resistant plasmodia strains. 